LETTER TO JMG

\title{
The M98K variant of the OPTINEURIN (OPTN) gene modifies initial intraocular pressure in patients with primary open angle glaucoma
}

\author{
R Melki, A Belmouden, O Akhayat, A Brézin, H-J Garchon
}

J Med Genet 2003;40:842-844

$\mathrm{P}$ rimary open angle glaucomas (POAGs) are the commonest form of glaucoma among Caucasians. They are defined by an excavation of the optic nerve head, a progressive loss of the visual field, and a normally open iridocorneal angle. ${ }^{1}$ Their prevalence is $1-2 \%$ and they are a major cause of irreversible blindness in Western countries. ${ }^{2}$ Albeit not necessary to the definition of POAG, an elevation of intraocular pressure (IOP) is a major contributory factor, and its lowering is at present the only available therapeutic strategy. In a subset of POAG patients, however, the IOP remains in the normal range-that is, less than $21 \mathrm{~mm} \mathrm{Hg}$, defining normal tension glaucoma (NTG). ${ }^{3}$ Whether NTG constitutes a pathological entity distinct from POAG is debated. ${ }^{4}$

Genetic factors play a major role in POAG predisposition. The genetic basis of POAG, is complex and heterogeneous. ${ }^{5}$ Recently, the OPTINEURIN (OPTN) gene on chromosome 10 pl4 was shown to be implicated in NTG. ${ }^{6}$ Mutations in this gene were found to segregate in nine out of 54 families with dominantly inherited POAG and normal or moderately elevated IOP. A variant, M98K, was associated with an increased absolute risk of $13.6 \%$ in a group of 169 POAG patients with normal or elevated IOP, compared with a relative allele frequency of $2.1 \%$ in a control population. ${ }^{6}$ This observation has made the M98K variant the potentially most important single genetic factor contributing to POAG predisposition. It is therefore essential to assess its role in different populations. In the present study, we evaluated the M98K variant in two groups of POAG patients, one from France and the other from Morocco, with population matched controls.

\section{PATIENTS AND METHODS}

Participants gave their informed consent, in keeping with European legislation and following a protocol reviewed and approved by supporting institutions. Both groups, 237 subjects from France and 56 from Morocco, had been previously described in detail $^{78}$; all were Caucasians. In France, they were recruited at the Glaucoma Institute of Saint-Joseph Hospital, the Quinze-Vingts Hospital in Paris, and the Lille University Hospital from 1994-1996. Moroccan patients were living in South Morocco, in the area of Agadir, Taroudant, and Ouarzazate, and were recruited from 19992000. Final POAG diagnosis was made at the time of inclusion, after review of inclusion and exclusion criteria (as previously described in detail) and before the genotyping was conducted. The initial IOP was defined as the maximal IOP known before initiation of treatment, usually at the time of diagnosis. All patients were unrelated. The French control group consisted of 110 healthy spouses from GLClA/MYOClinked glaucoma families. The Moroccan control sample comprised 60 age matched subjects from the general population of South Morocco.

\section{Key points}

- This work aimed to evaluate the M98K variant of the OPTINEURIN gene as a risk factor for primary open angle glaucoma (POAG) in participants from France $(\mathrm{n}=237)$ and from Morocco $(\mathrm{n}=56)$. The frequency of the variant was similar in patients and in matched control groups ( $n=110$ and $n=60$, respectively) in both populations.

- In the French patients, however, M98K was associated with a decreased initial intraocular pressure (IOP) (pressure difference $=0.0097$ ); in Moroccans the trend was not significant. The effect was again significant when both populations were pooled (pressure difference $=0.0052$ ). The M98K variant can be considered a modifier of the initial IOP in POAG patients.

Genotyping of M98K was performed by PCR-RFLP. The sequence of OPTN exon 5, which encodes this variant, was deduced from the cDNA sequence (Genebank accession number AF420370). Amplification of a 157 bp fragment was carried out with the following primers: forward FAM5'ggcctggacagagaaacaga $3^{\prime}$ and reverse 5'gctcacctcagat gacctttc 3', using reagents and conditions previously described, ${ }^{9}$ except that the annealing temperature was set at $62^{\circ} \mathrm{C}$. The $6603 \mathrm{~T}>\mathrm{A}$ variant, which encodes the M98K amino acid variation, creates an Stu I restriction site; cleavage of the variant polymerase chain reaction (PCR) product with Stu I generated two fragments, of 80 and $77 \mathrm{bp}$, which were detected after migration on an automated sequencer.

Allele frequencies were compared by assessing $2 \times 2$ tables. Distributions of IOP were compared with the nonparametric Wilcoxon-Mann-Whitney test. Pooled data were allowed for stratification according to the population. Exact $\mathrm{p}$ values were computed with the StatXact v5 software (Cytel, Cambridge, MA, USA). Because of prior knowledge of an association of M98K with NTG, one sided p values were considered. A similar analysis was also conducted after exclusion of patients who carried a disease causing mutation of the MYOCILIN gene.

\section{RESULTS}

Genotyping of the M98K variant of the OPTN gene was performed by PCR-RLFP, exploiting the creation of an Stu I

Abbreviations: POAG, primary open angle glaucoma; IOP, intraocular pressure; NTG, normal tension glaucoma; OPTN, OPTINEURIN; PCR, polymerase chain reaction 
Table 1 Frequencies of the M98K variant in POAG patients and in control subjects from France and Morocco

\begin{tabular}{lllll}
\hline Population & Status & Total & M98K (+) & $\%$ \\
\hline France & POAG & 237 & 11 & 4.64 \\
& Controls & 110 & 5 & 4.54 \\
Morocco & POAG & 56 & 6 & 10.7 \\
& Controls & 60 & 5 & 8.33 \\
\hline
\end{tabular}

POAG, primary open angle glaucoma.

restriction endonuclease site in the variant DNA. As shown in table 1, M98K frequencies in patients and in controls were similar, whether in France or in Morocco. However, there was a significant difference between the two populations, as the frequency of M98K was increased approximately twofold in Moroccan compared with French participants.

Since M98K was associated with glaucoma with normal or moderately elevated IOP, ${ }^{6}$ we investigated an influence of this variant on the initial IOP. There was no selection for or against NTG in the patient samples. The medians were $30 \mathrm{~mm} \mathrm{Hg}$ (range 14-70 mm Hg) in French patients and $36 \mathrm{~mm} \mathrm{Hg}$ (range 16-71 $\mathrm{mm} \mathrm{Hg}$ ) in Moroccan patients. As shown in table 2, there was a downward shift of the initial IOP in patients harbouring M98K, in both populations. This shift was significant in the French population only. Moreover, when both samples were pooled, the effect of M98K remained significant. This suggested that the trend observed in Moroccan patients could correspond to a weak effect.

The majority $(6 / 11,55 \%)$ of the French POAG patients positive for M98K belonged to the lower quartile of the distribution of initial IOP (values $<25 \mathrm{~mm} \mathrm{Hg}$ ), whereas none was present in the upper quartile of this distribution (values $>37 \mathrm{~mm} \mathrm{Hg}$ ). The odds ratio for M98K in the lower compared with the upper quartile was 9.03 (95\% confidence interval: $1.25-+\infty, \mathrm{p}=0.013$ ).

The above analysis was performed irrespective of the MYOCILIN gene mutation status. This was to account for the information actually available prior to any genotyping. As reported recently, 17 (7.5\%) French patients ${ }^{10}$ and one (3\%) Moroccan patient ${ }^{8}$ carried a mutation of the coding region of MYOC. In French patients, these mutations were associated with a significantly increased initial IOP. One of the French patients with a MYOC mutation, Q368X, was also positive for the M98K variant. Very interestingly, this double mutant patient had the lowest initial IOP, $23 \mathrm{~mm} \mathrm{Hg}$ at both eyes, among all MYOC mutation carriers. The other patients who were positive for M98K-that is, excluding the double mutant patient-had a significantly lower IOP than the MYOC mutation carriers (pressure difference $<1 \times 10^{-4}$ ). Moreover, when they were compared with the patients carrying a normal MYOC gene, the differences in IOP levels remained significant (table 2 ).

\section{DISCUSSION}

Our data indicate that M98K is not a risk factor for POAG by itself. However, at least among French POAG patients, M98K was associated positively with a low initial IOP and negatively with a high initial IOP. Therefore, it can be considered as a modifier of the glaucoma phenotype. This influence of the OPTN gene on IOP is consistent with its expression in the trabecular meshwork. ${ }^{6}$ Alternatively, as OPTN seems to interfere with induction of apoptosis, ${ }^{611}$ the M98K variant could increase the sensitivity of ganglial neural cells to risk factors of glaucoma. Consequently, in the presence of M98K, a lesser elevation of IOP would be required to damage the optic nerve.
Table 2 Decreased initial intraocular pressure in M98K+ patients: effect of removal of patients carrying a MYOCILIN mutation

\begin{tabular}{|c|c|c|c|}
\hline \multirow[b]{2}{*}{ MYOCILIN status $†$} & \multicolumn{2}{|c|}{ IOP $(\mathrm{mm} \mathrm{Hg})^{*}$} & \multirow[b]{2}{*}{ pł } \\
\hline & M98K§ & M98K§ & \\
\hline \multicolumn{4}{|l|}{ All cases } \\
\hline France & $25.9 \pm 3.6(11) \S$ & $32.3 \pm 9.5(179) \S$ & 0.0097 \\
\hline Morocco & $32.3 \pm 5.9(6)$ & $37.3 \pm 11.0(50)$ & NS \\
\hline Pooled & $28.2 \pm 5.4(17)$ & $33.4 \pm 10.1$ (229) & 0.0052 \\
\hline \multicolumn{4}{|l|}{ wt MYOC } \\
\hline France & $26.2 \pm 3.6(10)$ & $31.5 \pm 8.9(166)$ & 0.033 \\
\hline Morocco & $32.3 \pm 5.9(6)$ & $36.6 \pm 9.9(49)$ & NS \\
\hline Pooled & $28.5 \pm 5.4(16)$ & $32.7 \pm 9.4(215)$ & 0.017 \\
\hline
\end{tabular}

*Intraocular pressure at the time of diagnosis, before initiation of treatments.

tAll cases: all patients were considered, regardless of their MYOC mutation status; wt MYOC: only patients with a normal MYOC sequence were analysed.

\#Exact $p$ value of the Mann-Whitney test.

$\S M$ Mean \pm standard deviation (number of patients)

NS, not significant.

Interestingly, other clinical features were not altered by the presence of M98K. Thus, patients positive or negative for M98K did not differ at diagnosis in age, magnitude of cupping of the optic disc, severity of visual field loss or response to IOP lowering treatments (data not shown).

Despite its low frequency, the M98K variant appears to be present in different Caucasian populations. It will be interesting to investigate this variant in other ethnic groups and also its allelic associations with closely linked polymorphisms to test a common ancestry. The twofold difference observed for M98K prevalence between Moroccan and French populations nevertheless emphasises the importance of assessing the geographical origin of the patients and of matching the controls accordingly.

Additional studies will be necessary to evaluate the usefulness of the M98K variant for the clinical management of POAG patients. However, this variant is confirmed as an interesting marker for investigating the genetic heterogeneity of POAG and for testing phenotype-genotype correlations. It comes in addition to other recently described polymorphisms in the OPAl and APOE genes, which were associated with NTG or with modification of IOP in POAG patients. ${ }^{72}{ }^{13}$ Our data also warrant a detailed analysis of other OPTN gene polymorphisms in POAG patients.

\section{ACKNOWLEDGEMENTS}

We are grateful to participating patients. We thank Dr Jean-Claude Dascotte, Françoise Valtot, Prof. Alain Béchetoille, Dr Abderrahmane Idhajji, Dr Said Driouiche, Dr Moustapha Idrissi Hassani, and Abdelaziz Boukabboucha for providing access to clinical records of their patients.

This work was supported by INSERM (Paris, France), the FranceMaroc cooperation (CMIFM, AI-237/SVS/2000 and INSERMCNCPRST 2000), by the Ministère de l'Enseignement Supérieur du Maroc (PROTARS III, D14/56), and by the Assistance PubliqueHôpitaux de Paris (AOM96110).

\section{Authors' affiliations \\ R Melki, H-J Garchon, INSERM U580, Hôpital Necker, Paris, France R Melki, A Belmouden, O Akhayat, Laboratoire de Biologie Cellulaire et Moléculaire, Faculté des Sciences, Université Ibnou Zohr, Agadir, Morocco \\ A Brézin, Service d'Ophthalmologie, Hôpital Cochin, Paris, France}

Correspondence to: Dr H-J Garchon, INSERM U580, Hôpital Necker, 161 rue de Sèvres, 75743 Paris Cedex 15, France; garchon@necker.fr 


\section{REFERENCES}

1 Quigley HA. Open-angle glaucoma. N Engl J Med 1993;328:1097-106.

2 Quigley HA. Number of people with glaucoma worldwide. Br J Ophthalmol 1996:80:389-93.

3 Drance SM, Sweeney VP, Morgan RW, et al. Studies of factors involved in the production of low tension glaucoma. Arch Ophthalmol 1973;89:457-65.

4 Kamal D, Hitchings R. Normal tension glaucoma - a practical approach. Br J Ophthalmol 1998;82:835-40.

5 WuDunn D. Genetic basis of glaucoma. Curr Opin Ophthalmol 2002; 13:55-60.

6 Rezaie T, Child A, Hitchings R, et al. Adult-onset primary open-angle glaucoma caused by mutations in optineurin. Science 2002;295:1077-9.

7 Copin B, Brezin AP, Valtot $F$, et al. Apolipoprotein E-promoter singlenucleotide polymorphisms affect the phenotype of primary open-angle glaucoma and demonstrate interaction with the myocilin gene. Am J Hum Genet 2002;70:1575-81.
8 Melki R, Idhajii A, Driouiche S, et al. Mutational analysis of the MYOCILIN gene in patients with primary open-angle glaucoma in Morocco. Ophthalmic Genet 2003;24(3): 153-60.

9 Belmouden A, Melki R, Hamdani M, et al. A novel frameshift founder mutation in the cytochrome P450 1B1 (CYP1B1) gene is associated with primary congenital glaucoma in Morocco. Clin Genet 2002;62:334-9.

10 Melki R, Belmouden A, Brézin AP, et al. MYOCILIN analysis by DHPLC in French POAG patients: increased prevalence of Q368X mutation. Hum Mutat 2003;22(2):179.

11 Li Y, Kang J, Horwitz MS. Interaction of an adenovirus E3 14.7-kilodalton protein with a novel tumor necrosis factor alpha-inducible cellular protein containing leucine zipper domains. Mol Cell Biol 1998;18:1601-10.

12 Aung T, Ocaka L, Ebenezer ND, et al. A major marker for normal tension glaucoma: association with polymorphisms in the OPAl gene. Hum Genet 2002;110:52-6.

13 Vickers JC, Craig JE, Stankovich J, et al. The apolipoprotein epsilon4 gene is associated with elevated risk of normal tension glaucoma. Mol Vis 2002;8:389-93.

$\mathrm{ECHO}$

NOD2/CARD15 mutations do not affect early course of Crohn's disease

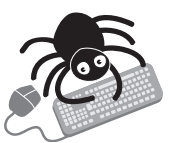

Please visit the Journal of Medical Genetics website [www. jmedgenet.com] for a link to the full text of this article.
E

uture searches for genetic markers of how Crohn's disease (CD) progresses will need to

stratify patients by disease aggressiveness, site, and smoking, as each influences the

early course of the disease. As importantly, NOD2/CARD15 genotype has no influence, according to the first study to use a validated classification of CD phenotype. Reliable predictors of disease course will mean patients receive optimal management early on.

The study looked for links between rapid progression of CD five years after diagnosis according to the Vienna classification and demographic, genetic, and disease variables.

Disease site and severity (number of flares/year) only were independent influences, according to multivariate analysis. Disease in the ileum significantly increased progression from initial non-stricturing, non-penetrating CD to stricturing CD five years later, and severity and smoking to progression to penetrating CD. For penetrating versus stricturing CD the markers were site and severity and familial disease. NOD2/CARD15 genotype bore no relation to disease state after five years. Overall, $68 \%$ of 163 patients with confirmed nonstricturing, non-penetrating CD initially had this disease pattern five years later, $11 \%$ had a stricturing form, and $22 \%$ a penetrating form.

Three mutations in the NOD2/CARD15 gene are linked to CD in Caucasians, but only in a quarter to a half of cases. CD is phenotypically very variable, and the stricturing and penetrating forms have more complications and need for operations. The investigators had already shown that the disease changed over time-from mostly non-structuring, nonpenetrating to structuring or penetrating some 25 years later.

A Gut 2003;52:552-557. 